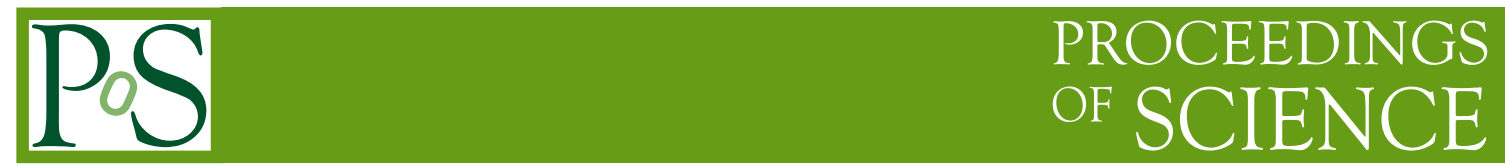

\title{
Recent Results from Super-Kamiokande
}

\author{
Masayuki Nakahata* for the Super-Kamiokande collaboration \\ Kamioka Observatory, Institute for Cosmic Ray Research, University of Tokyo \\ Kavli Institute for the Physics and Mathematics of the Universe (WPI), University of Tokyo \\ E-mail: nakahatalsuketto.icrr.u-tokyo.ac.jp
}

Recent results from the study of atmospheric neutrinos and solar neutrinos and from searches for sterile neutrinos and dark matter at Super-Kamiokande are presented. In addition, a feasibility study for dissolving gadolinium in the detector for a supernova relic neutrino measurement is presented.

XVI International Workshop on Neutrino Telescopes,

2-6 March 2015

Palazzo Franchetti ? Istituto Veneto, Venice, Italy

\footnotetext{
* Speaker.
} 


\section{Introduction}

Super-Kamiokande (SK) is a 50-kton water Cherenkov detector that has 11,000 20-inchdiameter photomultipliers (PMTs) in an inner detector and 1,885 8-inch-diameter PMTs in an outer detector[1]. Data collection by SK was started in April 1996, and it is now running the 'fourth phase' of its operation, begun in 2008. Oscillations of atmospheric and solar neutrinos were discovered at SK in 1998 and 2001, respectively. Since then, precise measurements of these neutrinos have been performed and various aspects of neutrino oscillations have been unraveled, such as the appearance of $v_{\tau}$ from atmospheric neutrinos [2] and the day/night difference of the solar neutrino flux [3]. SK has been obtaining data for nearly twenty years and is still the world's largest neutrino detector, covering an energy range from a few $\mathrm{MeV}$ to tens of $\mathrm{GeV}$. In this presentation, recent results from the study of atmospheric and solar neutrinos as well as new analyses on the searches for sterile neutrinos and dark matter are presented.

\section{Atmospheric Neutrinos}

Cosmic rays interact with air nuclei, producing showers of particles. The decay of secondary pions and kaons, created in the cosmic ray interactions, produce atmospheric neutrinos. Neutrinos travel 10-10,000 km before being detected. Both $v_{\mu}$ and $v_{e}$ are produced in the atmosphere. At lower energy, the ratio $v_{\mu} / v_{e}$ is almost two, which is determined by the decay scheme of pions. Both neutrinos and anti-neutrinos are produced, with $30 \%$ of observed data being anti-neutrinos, which is mainly determined by the difference in their cross sections. The flux of atmospheric neutrinos spans many decades in energy, $\sim 100 \mathrm{MeV}-100 \mathrm{TeV}$. Because of these characteristics, atmospheric neutrinos are an excellent tool for broad studies of neutrino oscillations.

Atmospheric neutrino oscillations are interpreted as $v_{\mu} \rightarrow v_{\tau}$ oscillations because of the deficit of $v_{\mu}$ and the lack of strong deviation from the expectation for $v_{e}$. Since the cross section of $v_{\tau}$ charged-current (CC) interaction is small, it has not been easy to obtain a signature of $v_{\tau}$. However, the long time-period observations of SK has enabled us to detect $v_{\tau}$.[2] We searched for events consistent with the hadronic decay of $\tau$ leptons. They are multi-ring electron-like events with visible energies above $1.3 \mathrm{GeV}$. The production of $v_{\tau}$ in cosmic ray interactions is negligible, so if $v_{\tau}$ are observed, they must be oscillation induced and must be upward-going events. A neural network (NN) method was adopted for this search and an $\mathrm{NN}$ variable was defined using the variables that characterize $\tau$ lepton decay. On a two-dimensional plane of the NN variable and the zenith angle $\left(\cos \left(\theta_{\text {zenith }}\right)\right)$, the obtained data were fitted with background and expected signal distributions. Figure 1 shows the zenith angle distribution of $\tau$-like events ( $\mathrm{NN}$ variable $>0.5$ ). A clear excess of $\tau$ signal is seen in the upward-going bins. The excess corresponds to the observed number of $180.1 \pm 44.3$ (stat.) $)_{15.2}^{17.8}$ (sys.) $\tau$-CC events, which is a $3.8 \sigma$ excess and $1.42 \pm 0.35$ times the expected number from the best fit oscillation parameters.

A study of the neutrino oscillation parameters of $\Delta m_{32}^{2}, \sin ^{2} \theta_{23}, \delta \mathrm{CP}$, and mass hierarchy was performed using 4581 days of data taken until 2014. More than 47,500 atmospheric neutrino events were observed during this time and the events are subdivided by event topology (fully-contained, partially-contained and upward-going $\mu$ ), energy range (sub-GeV and multi-GeV), $e$ - or $\mu$-like and the number of rings. Multi-GeV $e$-like samples are further subdivided into $v$-like and $\bar{v}$-like 


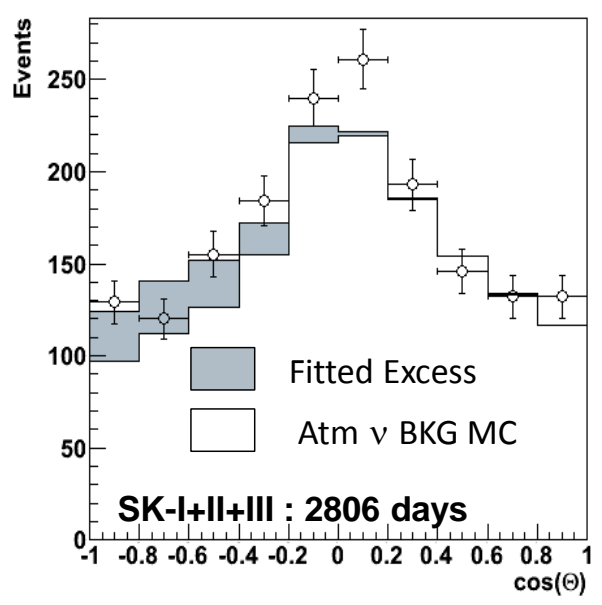

Figure 1: Zenith angle distribution of $\tau$-like events. The fitted $\tau$ signal and background from other atmospheric neutrino interactions are shown in gray and white, respectively.
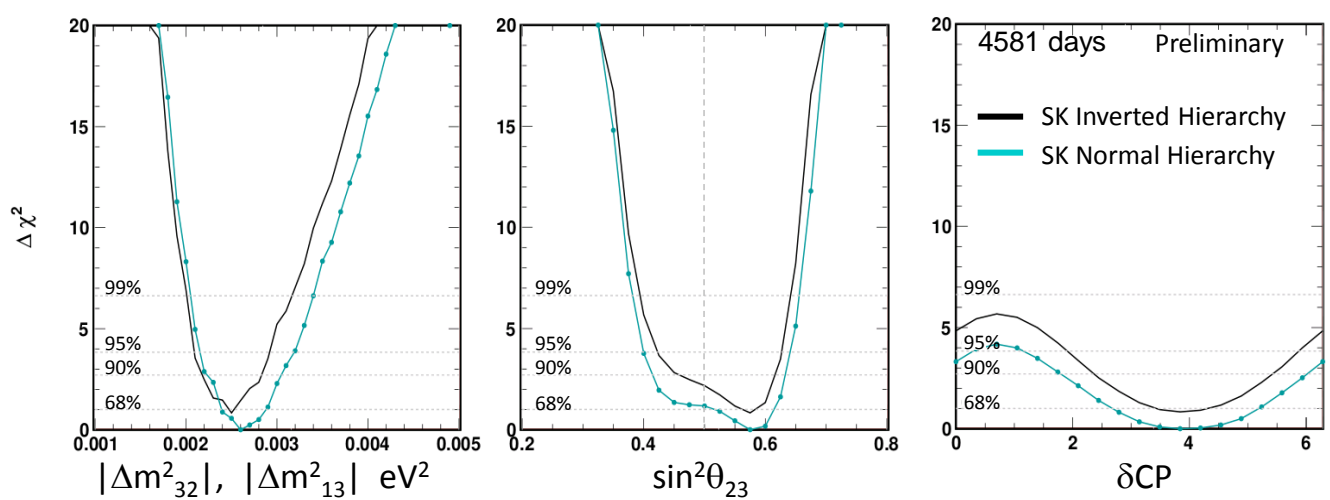

Figure 2: $\Delta \chi^{2}$ distribution of oscillation parameters obtained from SK data. The black and blue curves show inverted and normal hierarchies, respectively.

samples in order to discriminate the mass hierarchy. The obtained oscillation parameters using only SK data are shown in Fig.2. In this analysis, $\theta_{13}$ is fixed to the average value of the Particle Data Group (PDG), which is essentially determined by short baseline reactor experiments. The black and blue curves in the figure show the inverted and normal hierarchies, respectively, and their relative difference shows the preference of the mass hierarchy. The normal hierarchy is favored at the level of $\Delta \chi^{2}=0.9$. Two dimensional contours of $\left|\Delta m_{32}^{2}\right|$ and $\sin ^{2} \theta_{23}$ are shown in Fig.3 together with $\mathrm{T} 2 \mathrm{~K}$ results from $v_{\mu}$ disappearance data and MINOS beam+atmospheric $v$ data. T2K and MINOS give a better resolution for $\left|\Delta m_{32}^{2}\right|$ than SK atmospheric $v$, and T2K also gives a better measurement for $\sin ^{2} \theta_{23}$. It is therefore expected that an analysis of the SK atmospheric data constrained with the T2K data should give a better measurement of $\delta \mathrm{CP}$ and mass hierarchy. The plots in Figure 4 demonstrate this. The light blue curves are obtained from SK data constraining by the T2K data. This plot is for normal hierarchy and a similar plot is obtained for inverted hierarchy. The minimum $\chi^{2}$ of these cases were compared and the normal hierarchy is favored at the level of $\Delta \chi^{2}=1.2$. The range of disfavored $\delta C P$ increases, but $C P$ conservation $(\sin (\delta C P)=0)$ is still 

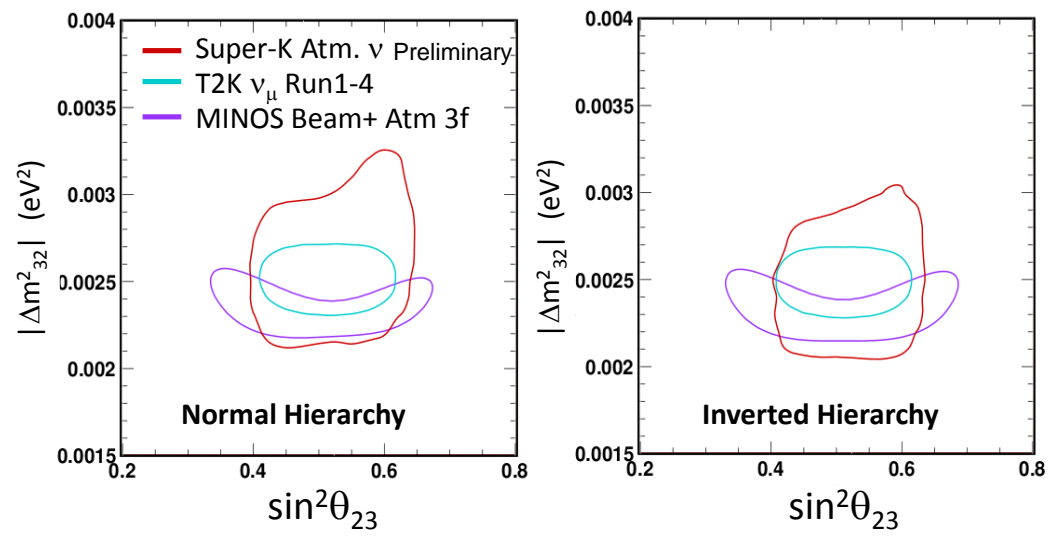

Figure 3: $90 \%$ contours of $\left|\Delta m_{32}^{2}\right|$ and $\sin ^{2} \theta_{23}$ obtained from SK atmospheric neutrino data (red), T2K $v_{\mu}$ disappearance data (light blue) and MINOS beam+atmospheric $v$ data (purple) for the normal (left) and inverted (right) hierarchies.
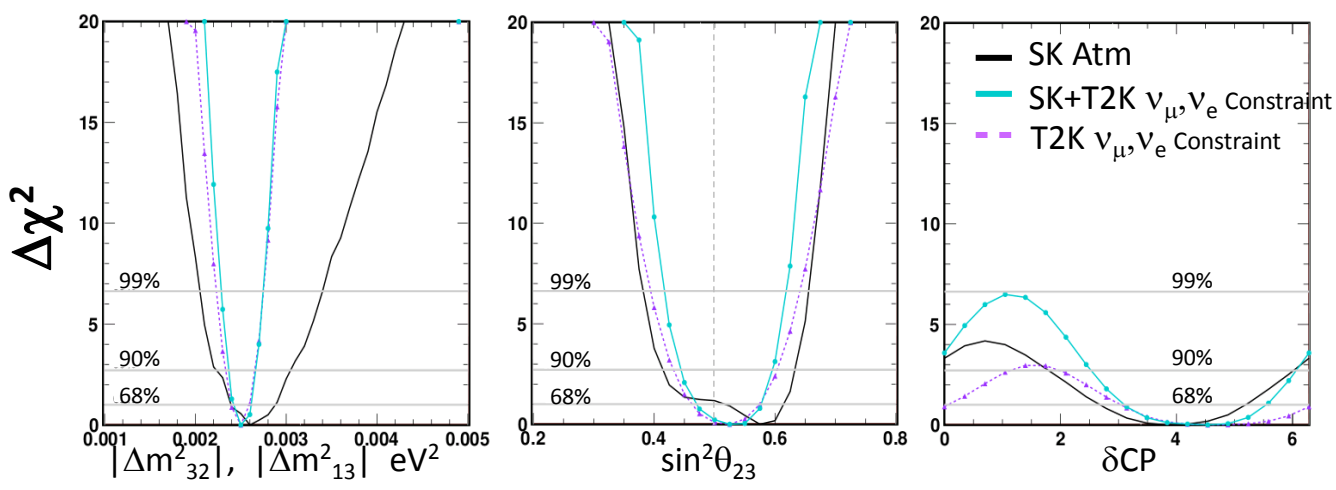

Figure 4: $\Delta \chi^{2}$ distribution of oscillation parameters obtained from SK atmospheric $v$ data (black), T2K data (purple dotted) and SK data constrained with T2K data (light blue). The normal hierarchy is assumed.

allowed at a C.L of at least $90 \%$ for both hierarchies.

The LSND experiment claimed a $\overline{v_{e}}$ appearance in a $\overline{v_{\mu}}$ beam consistent with $\Delta m^{2} \sim 1 \mathrm{eV}^{2}$ and the MiniBooNE experiment detected anomalies that may indicate oscillation of a similar $\Delta m^{2}$. In addition, it has been suggested that measurements of reactor experiments at short distance show a deficit in the $\bar{v}_{e}$ flux. Also, the rate of $v_{e}$ from ${ }^{51} \mathrm{Cr}$ and ${ }^{37} \mathrm{Ar}$ calibration sources in Gallium solar neutrino experiments (SAGE and GALLEX/GNO) was about $3 \sigma$ lower than the expectation. In order to reconcile these observations with established atmospheric and solar neutrino oscillations, an additional (fourth) neutrino (or more) is required. Since the LEP experiments measured the number of light neutrino families to be $2.980 \pm 0.0082$, the additional required neutrinos must be sterile. We have searched for sterile neutrinos which have $\mathrm{eV}^{2}$-scale $\Delta m^{2}$ from the 4438 live-days atmospheric neutrino data sample.[4] The standard neutrino mixing matrix, called the PontecorvoMaki-Nakagawa-Sakata (PMNS) matrix, was enlarged to include the fourth mass eigenstate and we searched for possible finite values of $\left|U_{\mu 4}\right|^{2}$ and $\left|U_{\tau 4}\right|^{2}$. If $\left|U_{\mu 4}\right|^{2}$ has a finite value, it induces a decrease in the event rate of $\mu$-like data for all energies and zenith angles. On the other hand, a finite value of $\left|U_{\tau 4}\right|^{2}$ induces a shape distortion of the zenith angle distributions for higher energy 

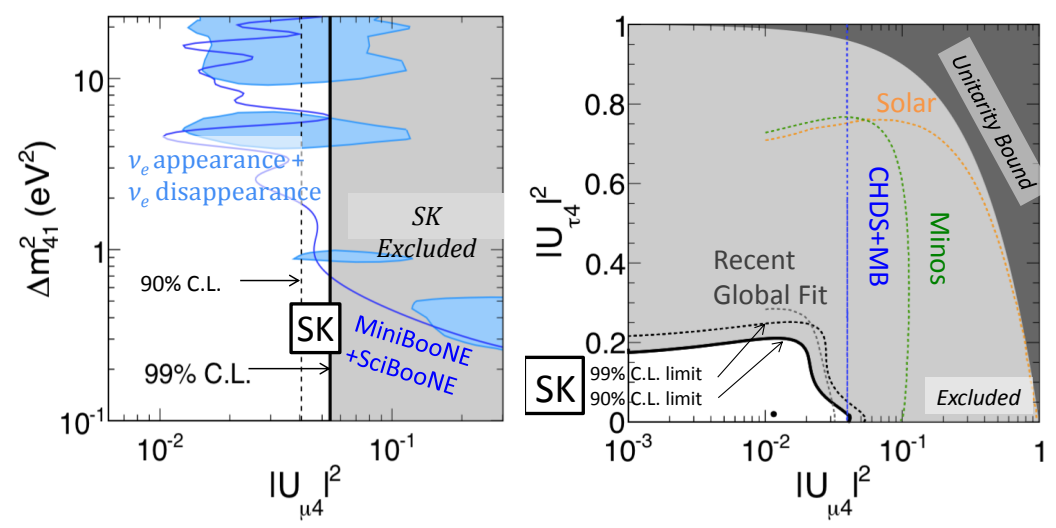

Figure 5: Limits on $\left|U_{\mu 4}\right|^{2}$ (left) and on $\left|U_{\mu 4}\right|^{2}$ and $\left|U_{\tau 4}\right|^{2}$ (right) obtained from 4438 live-days atmospheric neutrino data. The left figure also shows the allowed regions obtained by a combined analysis of $\bar{v}_{e}$ appearance and disappearance data from [5] and an excluded region by a MineBooNE and SciNooNE combined analysis from [6].

$\mu$-like data, e.g., a partially-contained through-going sample. The SK data can be reproduced using only the standard PMNS matrix framework and no indications of finite $\left|U_{\mu 4}\right|^{2}$ and $\left|U_{\tau 4}\right|^{2}$ were observed. A fully generic sterile oscillation model is difficult computationally because matter effects for both active $v_{e}$ and sterile neutrinos (neutral current (NC)) must be taken into account. Hence, we performed two different analyses. The first analysis took into account $v_{e}$ matter effects only and fitted only $\left|U_{\mu 4}\right|^{2}$. This method gave the most accurate $\left|U_{\mu 4}\right|^{2}$ limit and $\left|U_{\mu 4}\right|^{2}<0.041$ (90 \% C.L.) was obtained as shown in Fig.5(left). The SK limit excluded a fraction of the region allowed by the combined analysis of $\bar{v}_{e}$ appearance and disappearance data presented in [5]. The second analysis took into account NC matter effects only and fitted $\left|U_{\mu 4}\right|^{2}$ and $\left|U_{\tau 4}\right|^{2}$. The limit thus obtained is shown in Fig.5(right).

\section{Indirect Dark Matter Search}

A search for WIMPs by detecting neutrinos produced from WIMP annihilations occurring inside the Sun has been recently performed[7] using 3903 days of SK data taken until March 2012. In contrast with our old WIMPs analysis, which used only upward-going muons, the recent analysis used all categories (fully-contained, partially-contained and upward-going muons), all energies (sub-GeV and multi-GeV) and all flavors ( $e$-like and $\mu$-like). By including lower energy neutrinos, the recent analysis has improved the sensitivity for low mass WIMPs. The signature of the neutrinos from WIMP annihilations in the Sun is an excess of events in the direction of the Sun. Angular and reconstructed momentum distributions were fitted with the atmospheric neutrino background and possible signals from WIMPs. To simulate the neutrino flux from WIMP annihilation in the Sun, the WIMPSIM 3.01 simulator was used. We searched for three annihilation channels, $\chi \chi \rightarrow \tau^{+} \tau^{-} / b \bar{b} / W^{+} W^{-}$. No significant excess was observed and limits on neutrino fluxes were obtained. The flux limits were converted to an upper limit on WIMP-nucleon cross sections using DARKSUSY 5.0.6. Figure 6 shows the obtained limits on the WIMP mass vs. cross section plane for spin-dependent (SD) and spin-independent(SI) interactions. Thanks to the 

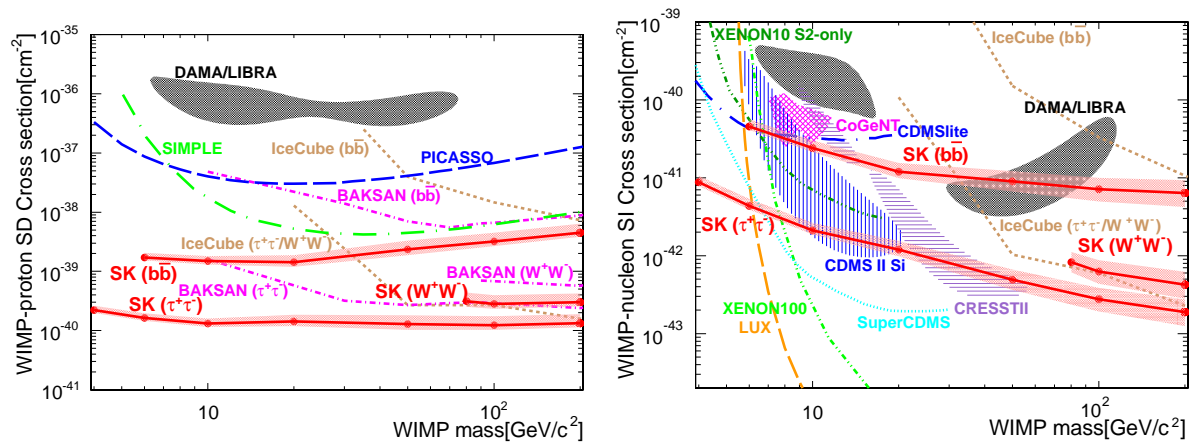

Figure 6: $90 \%$ C.L. upper limits on SD WIMP-proton (left) and SI WIMP-nucleon (right) cross sections obtained from 3903 days SK data. The default value calculated with DARKSUSY is shown as a red solid line and the bands show the range of uncertainties.

hydrogen-rich composition of the Sun, a tight limit on the SD cross section was obtained, and this SK analysis placed the most stringent constraint for WIMP masses below $200 \mathrm{GeV} / \mathrm{c}^{2}$ for the $\chi \chi \rightarrow \tau^{+} \tau^{-}$decay channel. For the limit on the SI cross section, this SK analysis excluded a new region for WIMP masses below $6 \mathrm{GeV} / \mathrm{c}^{2}$.

\section{Solar Neutrinos}

Because of the large fiducial mass of $22.5 \mathrm{kton}, \mathrm{SK}$ can observe ${ }^{8} \mathrm{~B}$ solar neutrinos with quite high statistics. The observed event rate at SK is about 20 events/day and we have observed about 70,000 signal events so far. Using this high statistical data, we have performed searches for time variation of the solar neutrino flux, energy spectrum distortion due to the oscillation effect as well as precise measurement of the oscillation parameters of $\Delta m_{21}^{2}$ and $\theta_{12}$. Figure 7 shows the yearly variation of the solar neutrino flux. The solar activity estimated from the sunspot number has changed over the last 18 years and 1.5 solar cycles have passed. However, no significant correlation between the solar neutrino flux and solar activity was observed. The $\chi^{2}$ of the SK data points for the flat distribution in Fig.7 is $\chi^{2}=13.53 / 17$ D.O.F., which corresponds to $70 \%$ probability. The flux ratio of the data to the standard solar model (SSM) prediction obtained from 4503 days of SK data taken until January 2014 is Data/SSM $=0.4463 \pm 0.0085$ (stat.+sys.), where the SSM is an un-oscillated flux of $5.25 \times 10^{6} / \mathrm{cm}^{2} / \mathrm{sec}$.

$v_{e}$ produced at the core of the Sun are oscillated into $v_{\mu}$ and $v_{\tau}$, and $v_{e}$ can be "re-generated" due to the matter effect through the Earth. This matter effect may cause a difference in the daytime and nighttime fluxes. Using the high statistical SK data, this day/night asymmetry was observed.[3] The time variation pattern during the night depends on oscillation parameters, especially $\Delta m_{21}^{2}$. Hence, the expected shape of the time variation was calculated and the amplitude of the variation was fitted using the extended maximum likelihood method. The obtained day/night asymmetry $\left(A_{D N} \equiv \frac{(\text { day }-n i g h t)}{(\text { day }+ \text { night }) / 2}\right)$ as a function of $\Delta m_{21}^{2}$ is shown in Fig.8 together with the expected $A_{D N}$. The $\Delta m_{21}^{2}$ range measured using KamLAND reactor neutrinos and the solar global analysis are also shown in the figure, and the observed $A_{D N}$ is consistent with the expectation within about the $1 \sigma$ level. The $A_{D N}$ value for $\Delta m_{21}^{2}=4.84 \times 10^{-5} \mathrm{eV}^{2}$ is $-3.3 \pm 1.0 \pm 0.5 \%$ and its non-zero significance is $3.0 \sigma$. 


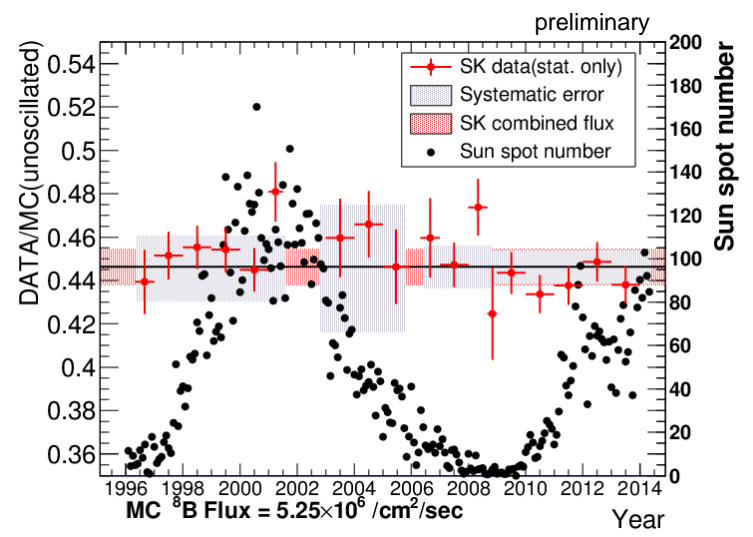

Figure 7: The red points with error bars show the yearly ${ }^{8} \mathrm{~B}$ flux obtained by SK. The vertical axis shows the relative ratio of data and an un-oscillated MC prediction with a flux of $5.25 \times 10^{6} / \mathrm{cm}^{2} / \mathrm{sec}$. The errors are statistical only. The gray bands show systematic errors for each SK phase. The pink band shows the SK combined flux taking into account statistical and systematic errors. The black dots show the time variation of sunspot numbers taken from [8].

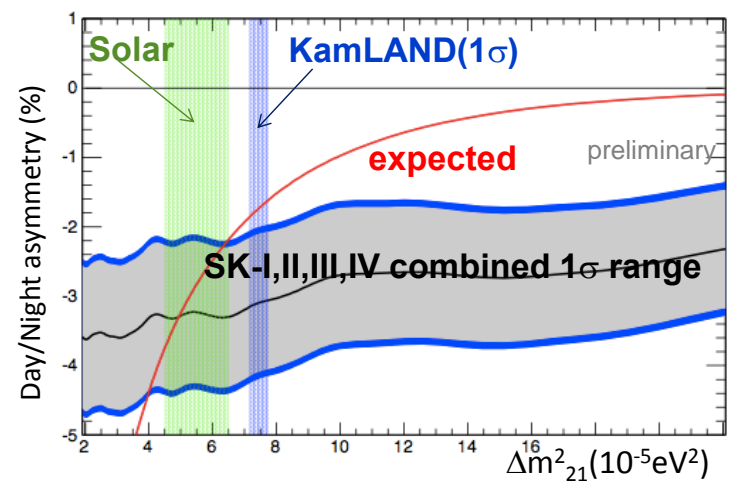

Figure 8: The band centered with the black curve shows the day/night asymmetry $\left(A_{D N}\right)$ of the SK solar neutrino flux as a function of $\Delta m_{21}^{2}$ with the statistical error in gray and the systematic error in blue. The red curve shows the expected value of $A_{D N}$. The vertical light blue and light green bands show the $\Delta m_{21}^{2}$ ranges measured using KamLAND reactor neutrinos and the solar global analysis, respectively.

The solar neutrino oscillations are "vacuum"-dominant and "matter"-dominant oscillations for lower and higher energy, respectively. Their oscillation probabilities are $P \simeq 1-0.5 \sin ^{2} 2 \theta \simeq 0.55$ and $P \simeq \sin ^{2} \theta \simeq 0.3$, respectively. Therefore, there must be a turning point, which is expected to be at a few MeV. We are currently searching for such an energy spectrum distortion at SK. Energy spectra were generated for each SK phase and the combined energy spectrum is shown in Fig.9. We have not yet observed any spectral distortion. The original neutrino spectrum was fitted with quadratic and exponential functions, and the resultant spectrum in SK is compared with the MSW expectations with solar+KamLAND and solar-global oscillation parameters. The MSW expectations are disfavored with a 1.0-1.7 $\sigma$ level.

Using the most up-to-date SK solar neutrino data as well as the latest results of other solar neutrino experiments, the allowed regions of the oscillation parameters of $\Delta m_{21}^{2}$ and $\theta_{12}$ are obtained as shown in Fig.10. In this analysis, $\sin ^{2} \theta_{13}$ is constrained to $0.0242 \pm 0.0026$, obtained from the short 


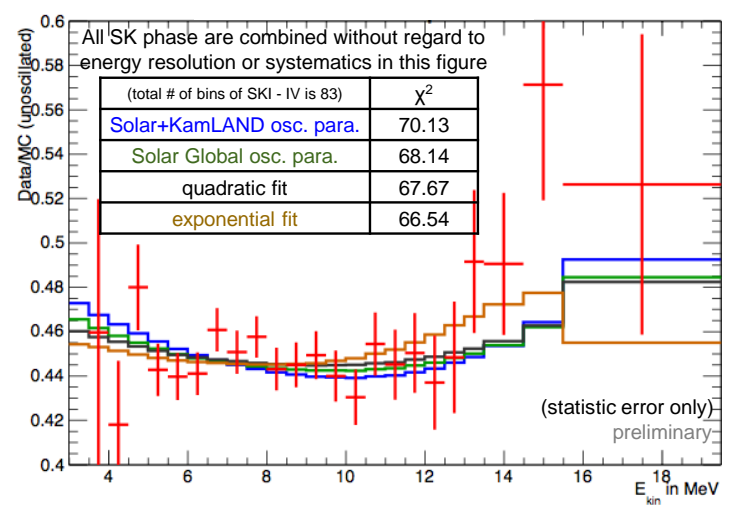

Figure 9: The red points with errors show the energy spectrum of solar neutrino data combining all SK phases. The vertical axis shows the ratio of the data to the un-oscillated SSM prediction with a flux of $5.25 \times 10^{6} / \mathrm{cm}^{2} / \mathrm{sec}$. The brown and black histograms show fits assuming exponential and quadratic energy dependent functions in neutrino oscillation probability, respectively. The blue and green histograms show the expectations for the oscillation parameters of global analyses of solar+KamLAND and solar-only, respectively.

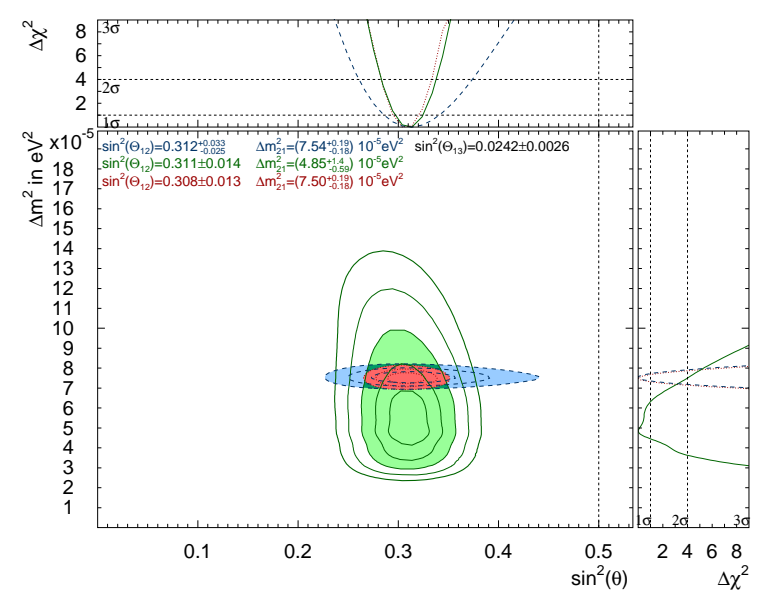

Figure 10: Allowed region of oscillation parameters of $\Delta m_{21}^{2}$ and $\theta_{12}$ obtained from solar global (green), KamLAND reactor (light blue) and solar+KamLAND(red). The best fit parameters and their $1 \sigma$ errors are also shown.

baseline reactor experiments. The projection of the two-dimensional contour onto the $\Delta m_{21}^{2}$ axis shows that there seems to be about $2 \sigma$ tension between the solar-global and KamLAND reactor data in $\Delta m_{21}^{2}$.

\section{R\&D for GADZOOKS!}

Supernovae have been occurring since the beginning of the universe, and the neutrinos from all past supernovae exist as diffuse neutrinos in our universe, called supernova relic neutrinos (SRNs). Since the SRNs have accumulated since the beginning of the universe, they can tell us about the star formation history of the universe. The expected event rate in this energy range is about 1.3-6.7 


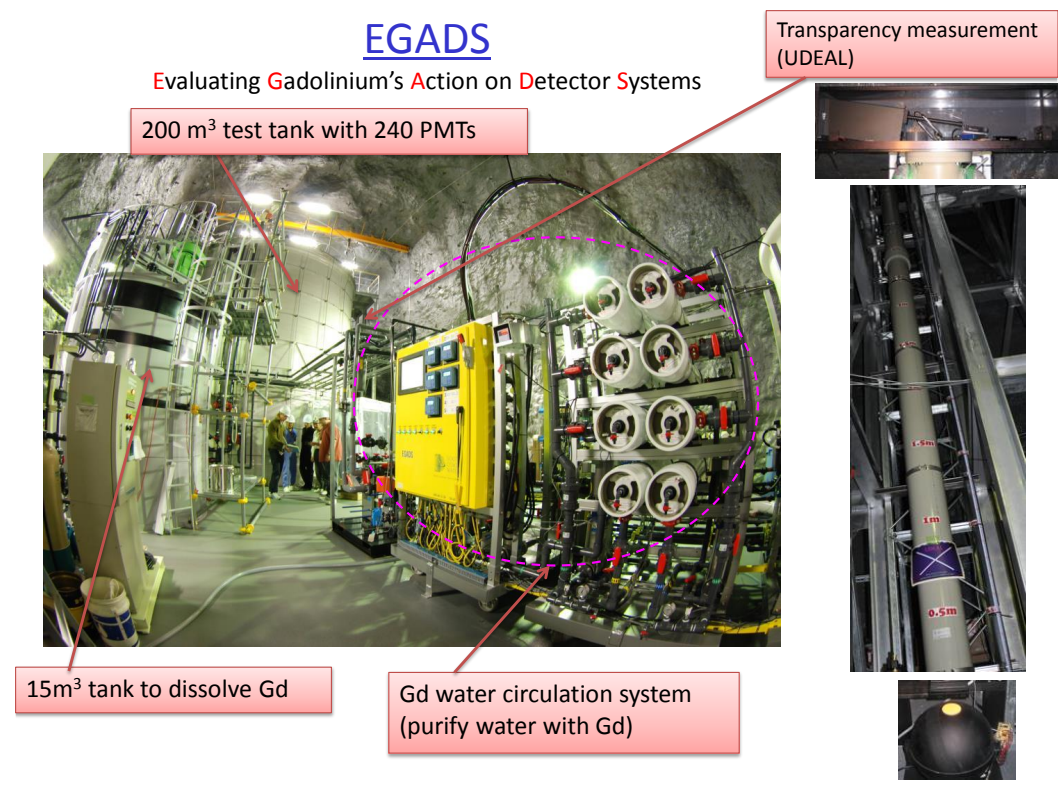

Figure 11: Equipment for EGADS: $15-\mathrm{m}^{3} \mathrm{Gd}$ premixing and pretreatment tank (left), selective filtration system (right), and 200- $\mathrm{m}^{3}$ tank (center).

events/year/22.5 kton. So, a large target mass like the SK detector and a new technique for high background reduction are necessary.

At present, the SK detector can detect only prompt positron signals. However, if it could detect delayed neutrons then the background constraining the SRN search would be greatly reduced. Such a reduction could be achieved via coincidence detection of positrons and neutrons (in space using vertex correlation of $\sim 50 \mathrm{~cm}$, and in time with $\sim 20 \mu \mathrm{s}$ ). By adding $0.2 \%$ gadolinium sulfate into the water tank, this goal can be achieved.[9] Gadolinium has a thermal neutron capture cross section of 49,000 barns (about five orders of magnitude larger than that of protons) and emits a gamma cascade at $8 \mathrm{MeV}$ that can be detected by Cherenkov light. In order to obtain a $90 \%$ efficiency for neutron capture, the Gd concentration should be $0.1 \%$, or $0.2 \%$ if $\mathrm{Gd}_{2}\left(\mathrm{SO}_{4}\right)_{3}$ is used.

In order to study the effect of dissolving Gd in the SK tank, an R\&D project called EGADS (evaluating gadolinium's action on detector systems) has been running in the Kamioka mine. A 200- $\mathrm{m}^{3}$ stainless steel tank which mimics the SK tank has been constructed (Fig. 11). The tank is equipped with a selective water filtration system that removes impurities while retaining the $\mathrm{Gd}$, a $15-\mathrm{m}^{3} \mathrm{Gd}$ premixing tank, a pretreatment system to remove radioactivity, and a device to measure the water attenuation length. In summer 2013, 240 PMTs were installed in the 200- $\mathrm{m}^{3}$ tank. Most are 20-inch PMTs of the same type used in the SK tank and a few PMTs are prototypes for Hyper-Kamiokande. The cabling and waterproofing methods are same as those used at SK.

Starting from November $2014, \mathrm{Gd}_{2}\left(\mathrm{SO}_{4}\right)_{3}$ was dissolved in pure water at $0.2 \%$ in the $200-\mathrm{m}^{3}$ tank. The transparency of the Gd-loaded water was evaluated from the intensity of Cherenkov light left at a distance of $15 \mathrm{~m}$, referred to as $L L_{15 \mathrm{~m}}$. The $L L_{15 \mathrm{~m}}$ value for the Gd-loaded water was measured to be better than $90 \%$. More detailed studies for possible effects on other SK physics are being performed. 


\section{Conclusions}

Recent results from the study of atmospheric and solar neutrinos as well as from searches for sterile neutrinos and dark matter at SK are presented. Using the large data sample accumulated at SK, neutrino oscillation parameters and the mass hierarchy have been studied. The normal hierarchy is favored at the level of $\Delta \chi^{2}=0.9$ using SK data alone and at $\Delta \chi^{2}=1.2$ with SK constrained by T2K $v_{e}$ and $v_{\mu}$ analyses. Additionally, recent progress in the analysis of atmospheric neutrinos is highlighted by evidence for the appearance of oscillation-induced $v_{\tau}$, which has been obtained with $3.8 \sigma$ significance. Further, limits on sterile neutrino oscillations have been obtained.

An indirect dark matter search has been performed using the atmospheric neutrino data sample. The expected signature is high energy neutrinos from the Sun, where WIMP self-annihilation produces secondary neutrinos that would appear on top of the atmospheric neutrino background. SK has placed the most stringent constraints on spin-dependent interactions on protons below 200 $\mathrm{GeV}$ and also set new limits on the spin-independent interactions of light WIMPs $(<6 \mathrm{GeV})$.

Additionally, SK has observed almost 70,000 ${ }^{8} \mathrm{~B}$ solar neutrino events during 4504 live-days, and the stability of the solar neutrino flux has been discussed with high precision. No significant correlation with the activity of the Sun has been observed even though the data-taking period spans almost 1.5 solar cycles. A difference between the daytime and nighttime solar neutrino fluxes, which is expected from the effects of matter on neutrino oscillations in the Earth, has been observed. However, no distortion of the energy spectrum, expected from solar neutrino oscillations, has yet been observed. The value of $\Delta m_{21}^{2}$ obtained from the SK solar neutrino data is tension with that from KamLAND reactor neutrino measurements by about $2 \sigma$.

In order to detect supernova relic neutrinos, the feasibility of dissolving gadolinium in the detector for enhanced neutron tagging was studied. A 200-ton test tank which mimics the SK detector was constructed and $0.2 \% \mathrm{Gd}_{2}\left(\mathrm{SO}_{4}\right)_{3}$ was dissolved within it. The Cherenkov light yield of the Gd-loaded water was measured to be better than $90 \%$ and more detailed studies are on-going.

\section{References}

[1] Super-Kamiokande collaboration (Y. Fukuda et al.), Nucl. Instrum. Meth. A501: 418-462 (2003).

[2] Super-Kamiokande collaboration (Y. Ashie et al.), Phys. Rev. Lett. 110, 181802 (2013).

[3] Super-Kamiokande collaboration (A. Renshaw et al.), Phys. Rev. Lett. 112, 091805 (2014).

[4] K. Abe et al., Phys. Rev. D91, 052019 (2015).

[5] J. Kopp et al., JHEP 1305(2013)050.

[6] G. Cheng et al., Phys. Rev. D86, 052009 (2012).

[7] K. Choi et al., Phys. Rev. Lett. 114, 141301 (2015).

[8] http://solarscience.msfc.nasa.gov/greenwch/spot_num.txt

[9] J. Beacom and M. Vagins, Phys. Rev. Lett. 93, 171101 (2004). 